\title{
GENERAL FINSLER CONNECTIONS ON A FINSLER VECTOR BUNDLE
}

\author{
By Aurel Bejancu AND TOMINOSUKe OTSUKI
}

\section{$\S 1$. Introduction.}

In the present paper, we shall describe a fundamental concept of general Finsler connections combining vectorial Finsler connections introduced by the first author for Finsler vector bundles [2] with general connections introduced by the second author for differentiable manifolds [7] and [8] and treat some special examples.

\section{§2. General Finsler connections on a Finsler vector bundle.}

Let $M$ be a real differentiable manifold of dimension $n$. Denote by $T M$ the tangent bundle over $M$ and by $\pi$ the canonical projection of $T M$ to $M$. Also, denote by $d \pi: T \backslash M \rightarrow T M$ the differential of $\pi$ and define the vertical subbundle $V T M$ of $T T M$ as the kernel of $d \pi$. A complementary distribution $N$ to VTM in TTM is called a non-linear connection on $T M$ (see M. Matsumoto [4] p. 55). Of course, the fibres of vector bundles $V T M$ and $N$ are of the same dimension $n$. In this section we have the following range of indices: $i, j, k, \cdots=1,2, \cdots, n$; $a, b, c, \cdots=1,2, \cdots, p$.

In order to get a local feature of the notion of non-linear connection we consider a canonical chart $\left(\mathcal{U}, \varphi, R^{2 n}\right)$ on $T M$, where $U$ is an open set of $T M$ and $\varphi: U \rightarrow R^{2 n}$ is a diffeomorphism of $U$ on $\varphi(U)$. For each $z \in \mathcal{U}$ denote by $\left(x^{1}, \cdots, x^{n} ; y^{1}, \cdots, y^{n}\right)$ its coordinates in this chart. We take another local chart $\left(\varphi^{\prime}, \mathcal{U}^{\prime}, R^{2 n}\right)$ on $T M$ such that $U \cap \mathcal{U}^{\prime} \neq \phi$. Then the non-linear connection $N$ is given by $n^{2}$ differentable functions $N^{i}$, on each $U$ satisfying

$$
N^{i^{\prime}}{ }_{j^{\prime}}=\frac{\partial x^{i^{\prime}}}{\partial x^{2}}\left(\frac{\partial x^{j}}{\partial x^{j^{\prime}}} N^{i}{ }_{j}+\frac{\partial^{2} x^{\imath}}{\partial x^{j^{\prime}} \partial x^{k^{\prime}}} y^{k^{\prime}}\right)
$$

at all points of $U \cap \mathcal{U}^{\prime}$, where $N^{i^{\prime}}{ }_{j^{\prime}}$ are the corresponding functions on $\mathcal{U}^{\prime}$. We denote by $\left\{\frac{\partial}{\partial x^{2}}, \frac{\partial}{\partial y^{2}}\right\}$ the natural local field of frames on $T M$. Then $\left\{\frac{\delta}{\delta x^{2}}\right\}$ given by

Received October 21, 1986 


$$
\frac{\delta}{\delta x^{i}}=\frac{\partial}{\partial x^{2}}-N^{j}{ }_{i} \frac{\partial}{\partial y^{2}},
$$

is a local field of frames on the distribution $N$.

Now let $\pi_{E}: E \rightarrow T M$ be a vector bundle over $T M$ with standard fibre $R^{p}$ and $\Gamma(E)$ be the module of all differentiable cross-sections of $E$. Then we call $E$ a Finsler vector bundle on $M$. A justification of the above name is the simple fact that all cross-sections of $E$ are depending on both, point and direction on $M$. The vertical subbundle VTM and the non-linear connection $N$ defined above are certainly Finsler vector bundles. Further, we consider a vector bundle morphism $P: E \rightarrow E$ and a general connection $\nabla$ (Otsuki connection) on $E$ with respect to $P$ or a general connection with $P$ as its component of the first order (see T. Otsuki [8] and [9]). Then by a recent work of $N$. Abe [1], a general connection on $T M$ is a mapping $\nabla: \Gamma(T T M) \times \Gamma(E) \rightarrow \Gamma(E)$ satisfying

$$
\begin{aligned}
& \nabla_{f X+Y} S=f \nabla_{X} S+\nabla_{Y} S \text { and } \\
& \nabla_{X}\left(f S+S^{\prime}\right)=(X f) P S+f \nabla_{X} S+\nabla_{X} S^{\prime},
\end{aligned}
$$

for any $S, S^{\prime} \in \Gamma(E), X, Y \in \Gamma(T T M)$ and differentiable function $f$ on $T M$.

We have now all the elements in order to introduce a new concept in Finsler geometry. We say that the triplet $(N, P, \nabla)$ is defining a general Finsler connection on the Finsler vector bundle $E$. If in particular, $P$ is the identity morphism $I_{E}$ on $E$, the general connection on $E$ is just a linear connection on $E$ and we obtain the concept of vectorial Finsler connection on $E$ (see A. Bejancu $[2])$.

Since as it is well-known in Finsler geometry, local computations are always welcome, we give further the local alternative of the definition of a general Finsler connection on $E$. In order to do this we consider a vectorial chart $\left(\Phi, \mathcal{U}, R^{p}\right)$ on the vector bundle $E$, where $U$ is an open set of $T M$ and $\Phi: \pi_{E^{-1}}$ $\rightarrow U \times R^{p}$ is a diffeomorphism. Let $\left(\Phi^{\prime}, U^{\prime}, R^{p}\right)$ be another vectorial chart such that $U \cap \mathcal{U}^{\prime} \neq \phi$. Denote by $\left\{S_{a}\right\}$ (resp. $S_{a^{\prime}}$ ) the local basis of differentiable sections of $E$ on $U$ (resp. $U^{\prime}$ ). Then there exist $p^{2}$ real differentiable functions $G_{a}^{a}$, defined on $\mathcal{U} \cap \mathcal{U}^{\prime}$ and satisfying

$$
S_{a^{\prime}}=G_{a^{\prime}}^{a} S_{a} .
$$

On the other hand, the addapted field of frames $\left\{\frac{\delta}{\delta x^{2}}, \frac{\partial}{\partial y^{2}}\right\}$ and $\left\{\frac{\delta}{\delta x^{i^{\prime}}}, \frac{\partial}{\partial y^{i^{\prime}}}\right\}$ on $U$ and $U^{\prime}$ respectively satisfy

$$
\frac{\delta}{\delta x^{i^{\prime}}}=\frac{\partial x^{i}}{\partial x^{i^{\prime}}} \frac{\delta}{\delta x^{2}} ; \quad \frac{\delta}{\delta y^{i^{\prime}}}=\frac{\partial x^{2}}{\partial x^{i^{\prime}}} \frac{\partial}{\partial y^{2}}
$$

Next, we define on each domain $U \subset T M$ and $\pi_{E}^{-1}(\mathcal{U})$ the local chart of $T M$ and the local vectorial chart of $E$, the differentiable functions $F_{a}{ }^{b}{ }_{\imath}$ and $C_{a}{ }^{b}$ by

$$
\nabla_{\delta / \delta x \imath} S_{a}=F_{a}{ }^{b} S_{b} ; \quad \nabla_{\partial / \partial y} S_{a}=C_{a}{ }^{b}{ }_{\imath} S_{b} .
$$


Thus, given a general Finsler connection on $E$ we have the quadruplet of differentiable functions $\left(N^{i}{ }_{j}, F_{a}{ }_{\imath}{ }_{\imath}, C_{a}{ }_{\imath}{ }_{\imath}, P_{a}^{b}\right)$ on each open set $U \subset T M$. Conversely, suppose on each domain $U \subset T M$ there exists a quadruplet of differentiable functions $\left(N^{i}{ }_{j}, F_{a}{ }^{b}, C_{a}{ }^{b}, P_{a}^{b}\right)$ satisfying (2.1) and

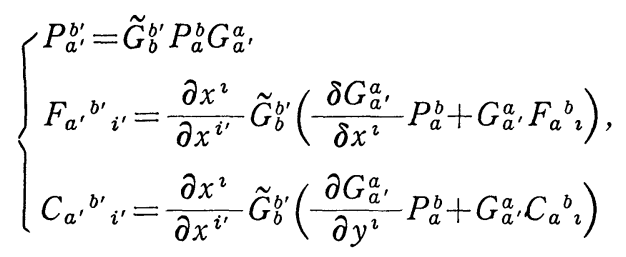

in each point of $U \cap U^{\prime}$, where $\left(N^{i^{\prime}}{ }_{j^{\prime}}, F_{a^{\prime}}{ }^{b^{\prime}}{ }_{i^{\prime}}, C_{a^{\prime}}{ }^{b^{\prime}}{ }_{i^{\prime}}, P_{a^{\prime}}^{b^{\prime}}\right)$ is the quadruplet on $U^{\prime}$ and $\left(\widetilde{G}_{b}^{b^{\prime}}\right)$ is the inverse matrix of $\left(G_{a^{\prime}}^{a}\right)$. Then it is routine to show by means of (2.1) and (2.8) the existence of a general Finsler connection on $E$.

Thus we have

THEOREM 2.1. Let $E$ be a Finsler vector bundle on $M$. Then the existence of a general Finsler connection on $E$ is equivalent to the existence of a quadruplet of differentiable functions $\left(N^{i}{ }_{j}, F_{a}{ }^{b}, C_{a}{ }^{b}, P_{a}^{b}\right)$ on each domain $U$ of $T M$ satisfying (2.1) and (2.8).

Let $G F C=(N, P, \nabla)$ be a general Finsler connection on the Finsler vector bundle $E$. Then the curvature tensor $\widetilde{R}$ of $\nabla$ is given by (see N. Abe [1])

$$
\begin{aligned}
\tilde{R}(X, Y) V= & \nabla_{X} \nabla_{Y} P V-\nabla_{Y} \nabla_{X} P V-P\left(\nabla_{[X, Y]} P V\right) \\
& -\left(\nabla_{X} I_{E}\right) \nabla_{Y} V+\left(\nabla_{Y} I_{E}\right) \nabla_{X} V,
\end{aligned}
$$

for any $X, Y \in \Gamma(T T M)$ and $V \in \Gamma(E)$. Taking into account that in Finsler geometry people is accustomed to three local curvature tensors we define the local components $R_{a}{ }_{\imath \jmath}, P_{a}{ }^{b}$, and $S_{a}{ }^{b}{ }_{\imath \jmath}$ of $\widetilde{R}$ by

$$
\left\{\begin{array}{l}
\tilde{R}\left(\frac{\delta}{\delta x^{j}}, \frac{\delta}{\delta x^{2}}\right) S_{a}=R_{a}{ }^{b}{ }_{2} S_{b} ; \quad \tilde{R}\left(\frac{\partial}{\partial y^{\jmath}}, \frac{\delta}{\delta x^{2}}\right) S_{a}=P_{a}{ }^{b}{ }_{2} S_{b} ; \\
\tilde{R}\left(\frac{\partial}{\partial y^{j}}, \frac{\partial}{\partial y^{2}}\right) S_{a}=S_{a}{ }^{b}{ }_{2 \jmath} S_{b} .
\end{array}\right.
$$

Then we denote the covariant components $\Lambda_{a}{ }^{b}{ }_{2}, \chi_{a}{ }^{b}{ }$ of the general Finsler connection $\nabla$, the components $I_{a}{ }^{b}{ }_{\imath}, J_{a}{ }^{b}{ }_{2}$ of $\nabla I_{E}$ with respect to $\delta / \delta x^{\imath}$ and $\partial / \partial y^{\imath}$ respectively and the components of the torsion tensor of $N$ as follows:

$$
\begin{aligned}
& \Lambda_{a}{ }^{b}{ }_{\imath}=F_{a}{ }^{b}{ }_{i}-\frac{\delta P_{a}^{b}}{\delta x^{2}} ; \quad I_{a}{ }^{b}{ }=F_{c}{ }^{b}{ }_{i} P_{a}^{c}-P_{c}^{b} \Lambda_{a}{ }^{c}{ }, \\
& \chi_{a}{ }^{b}{ }_{i}=C_{a}{ }^{b}{ }_{i}-\frac{\partial P_{a}^{b}}{\partial y^{2}} ; \quad J_{a}{ }^{b}{ }_{i}=C_{c}{ }^{b}{ }_{i} P_{a}^{c}-P_{c}{ }_{c} \chi_{a}{ }^{c}{ }_{i}, \\
& R^{k}{ }_{i j}=\frac{\delta N^{k}{ }_{i}}{\delta x^{j}}-\frac{\delta N^{k}{ }_{j}}{\delta x^{i}} .
\end{aligned}
$$


Thus by using (2.9)-(2.11) we obtain (after a long computation) the local components of curvature tensor field $\widetilde{R}$ given by

$$
\begin{aligned}
& R_{a}{ }^{b}{ }_{\imath j}=\left(\frac{\delta F_{c}{ }^{d}{ }_{i}}{\delta x^{j}}-\frac{\delta F_{c}{ }^{d}{ }_{j}}{\delta x^{2}}\right) P_{a}^{c} P_{d}^{b}+\left(F_{c}{ }^{d}{ }_{i} F_{d}{ }^{b}{ }_{j}-F_{c}{ }^{d}{ }_{j} F_{d}{ }^{b}{ }^{b}\right) P_{a}^{c} \\
& +I_{c}{ }_{i} \Lambda_{a}{ }_{a}{ }_{j}-I_{c}{ }_{j}{ }_{j} \Lambda_{a}{ }_{i}+R^{k}{ }_{2 j} C_{c}{ }^{d}{ }_{k} P_{a}^{c} P_{d}^{b}, \\
& P_{a}{ }^{b}{ }_{i j}=\left(\frac{\partial F_{c}{ }^{d}{ }_{i}}{\partial y^{j}}-\frac{\delta C_{c}{ }^{d}{ }_{j}}{\delta x^{2}}\right) P_{a}^{c} P_{d}^{b}+\left(F_{c}{ }^{d}{ }_{i} C_{d}{ }^{b}{ }_{j}-C_{c}{ }^{d}{ }_{j} F_{d}{ }^{b}{ }_{i}\right) P_{a}^{c} \\
& +I_{c}{ }_{i}{ }_{i} \chi_{a}{ }^{c}{ }_{j}-J_{c}{ }^{b}, \Lambda_{a}{ }^{c}{ }_{i}+\frac{\partial N^{k}{ }_{i}}{\partial y^{j}} C_{c}{ }^{d}{ }_{k} P_{a}^{c} P_{d}^{b}, \\
& S_{a^{b}{ }^{b} j}=\left(\frac{\partial C_{c}{ }^{d}{ }_{i}}{\partial y^{j}}-\frac{\partial C_{c}{ }^{d}{ }_{j}}{\partial y^{2}}\right) P_{a}^{c} P_{d}^{b}+\left(C_{c}{ }^{d}{ }_{i} C_{d}{ }^{b}{ }_{j}-C_{c}{ }^{d}{ }_{j} C_{d}{ }^{b}{ }_{i}\right) P_{a}^{c} \\
& +J_{c}^{b}{ }_{i}^{b} \chi_{a}{ }^{c}{ }_{j}-J_{c}{ }^{b}{ }_{j} \chi_{a}{ }^{c}{ }_{\imath} \text {. }
\end{aligned}
$$

It is easy to check that for the particular case $P=I_{E}$ from $(2.12)-(2.14)$ we obtain the local components of the curvature tensor of a vectorial Finsler connection on $E$ (see A. Bejancu [2]).

\section{§3. General Finsler connections induced by vectorial Finsler connections.}

Let $\pi_{E}: E \rightarrow T M$ and $\pi_{F}: F \rightarrow T M$ be two Finsler vector bundles on $M$. Suppose $V F C=(N, \nabla)$ is a vectorial Finsler connection on $E$. Then we consider the Whitney sum $G=E \oplus F$ and denote by $P$ the projection morphism from $G$ to $E$. Then we can define a general connection $\tilde{\nabla}$ on the whole $G$ with respect to $P$ by the following formula

$$
\tilde{\nabla}_{X} V=\nabla_{X} P V \quad \text { for } X \in \Gamma(T T M) \text { and } V \in \Gamma(G) \text {. }
$$

It is easy to verify that (3.1) defines a general connection on $G$. Thus, starting from the vectorial Finsler connection $\operatorname{VFC}=(N, \nabla)$ on $E$ we obtain on each Whitney sum $G$ of $E$ with any other Finsler vector bundle $F$, a general Finsler connection $G F C=(N, P, \tilde{\nabla})$ where $\tilde{\nabla}$ is given by (3.1). We call $G F C$ the induced general Finsler connection by the vectorial Finsler connection VFC. Denote by $\tilde{R}$ and $R$ the curvature tensors of $\tilde{\nabla}$ and $\nabla$ respectively. Then the following theorem seems to us very important.

THEOREM 3.1. The restriction of the curvature tensor of GFC to $E$ is just the curvature tensor $V F C$, that is, we have

$$
\tilde{R}(X, Y) V=R(X, Y) V \quad \text { for any } X, Y \in \Gamma(T T M), \quad V \in \Gamma(E) .
$$

Proof. First by a result of N. Abe [1] and (3.1) we have 


$$
\begin{aligned}
&\left(\tilde{\nabla}_{X} I_{G}\right)\left(\tilde{\nabla}_{Y} V\right)=\tilde{\nabla}_{X}\left(P \tilde{\nabla}_{Y} V\right)-P\left(\tilde{\nabla}_{X} \tilde{\nabla}_{X} V\right) \\
&=\nabla_{X}\left(P \nabla_{Y} P V\right)-P\left(\nabla_{X} P \nabla_{Y} P V\right) \quad \text { for any } \quad X, Y \in \Gamma(T T M) \\
& \text { and } V \in \Gamma(E) .
\end{aligned}
$$

But $P V=V$ and $P \nabla_{X} V=\nabla_{X} V$ since $\nabla_{X} V \in \Gamma(E)$. Hence by (3.3) we get

$$
\left(\tilde{\nabla}_{X} I_{E}\right)\left(\tilde{\nabla}_{Y} V\right)=0 \text {. }
$$

Thus, taking account of (3.4) in (2.9) we get our assertion.

Now, let $F^{n}=(M, F(x, y))$ be a Finsler space, where $M$ is an $n$-dimensional differentiable manifold and $F(x, y)$ is the fundamental function of $F^{n}$ (see M. Matsumoto [4]). Denote by $g_{\imath j}(x, y)$ the fundamental tensor field of $F^{n}$, that is we have

$$
g_{\imath j}(x, y)=\frac{1}{2} \frac{\partial^{2} F^{2}}{\partial y^{i} \partial y^{\jmath}} .
$$

Suppose $g_{\imath j}(x, y)$ is positive definite, that is, it defines a Riemannian metric on the vectorial bundle VTM. Next we consider a Finsler connection $F C$ on $F^{n}$ which in fact is just a vectorial Finsler connection $F C=(N, \nabla)$ on $V T M$, (see A. Bejancu [2], [3]). The existence of a non linear connection $N$ on $T M$ implies $T T M=N \oplus V T M$. Hence by the above construction we get a general Finsler connection $V G F C=(N, v, \tilde{\nabla}), \tilde{\nabla}$ is given by (3.1) and $v$ is the projection morphism of TTM to VTM. We call VGFC the vertical general Finsler connection on TTM.

Now we define the isomorphism of vector bundles

$$
L: N \rightarrow V T M \quad \text { by } L(X)=X^{i} \frac{\partial}{\partial y^{2}}, \text { where } X=X^{i} \frac{\delta}{\delta x^{i}} .
$$

It is easy to check that $L$ does not depend on the local coordinates $\left(x^{2}, y^{i}\right)$ on $T M$. Then we define a linear connection $\nabla^{*}$ on the vector bundle $N$ by

$$
\nabla_{X}^{*} U=L^{-1}\left(\nabla_{X} L U\right) \quad \text { for any } \quad X \in \Gamma(T T M), \quad U \in \Gamma(N) .
$$

Next, denote by $\tilde{\nabla}^{*}$ the general connection induced by $\nabla^{*}$ on TTM. Hence we have

$$
\tilde{\nabla}_{X}^{*} Y=\nabla_{X}^{*} h Y \quad \text { for any } \quad X, Y \in \Gamma(T T M(,
$$

where $h$ is the projection morphism of TTM to $N$. Thus we obtain another general Finsler connection $H G F C=\left(N, h, \tilde{\nabla}^{*}\right)$, whose curvature $\tilde{R}^{*}$ restricted to $N$ is just the curvature tensor $R^{*}$ of $\nabla^{*}$. We call HGFC the horizontal general Finsler connection on TTM.

As it is well-known, a Finsler connection $F C=\left(N^{i}{ }_{j}, F_{\jmath k}{ }^{2}, C_{\jmath k}{ }^{2}\right)$ on $F^{n}$ has five torsions $T_{j k}{ }^{2}, R_{j k}, P_{j k}^{\imath}, S_{j k}^{i}$ and $C_{j k}{ }^{\imath}$ given by

$$
T_{\jmath{ }_{k}}{ }^{2}=F_{\jmath}{ }_{k}-F_{k}{ }_{\jmath}{ }_{j} \text {; }
$$




$$
\begin{aligned}
& R^{i}{ }_{j k}=\frac{\delta N^{i}{ }_{j}}{\delta x^{k}}-\frac{\delta N^{i}{ }_{k}}{\delta x^{j}} ; \\
& P^{\imath}{ }_{j k}=\frac{\partial N^{i}{ }_{j}}{\partial y^{k}}-F_{k}{ }^{2}{ }_{j} ; \\
& S^{2}{ }_{j k}=C_{j}{ }_{k}-C_{k}{ }^{2} \text {; } \\
& \nabla_{\partial / \partial y^{i}} \frac{\partial}{\partial y^{j}}=C_{j}{ }^{k} i \frac{\partial}{\partial y^{k}} .
\end{aligned}
$$

On the other hand, for any general connection on TTM we can define its torsion tensor field (see T. Otsuki [7] and H. Nemoto [6]). Then we have

THEOREM 3.2. The torsion tensors of the horizontal and vertical general Finsler connections on TTM give all the torsions of the initial Finsler connection on $F^{n}$.

Proof. First, we denote by $T$ the torsion tensor field of the general connection $\tilde{\nabla}$. Thus we have

$$
T(X, Y)=\tilde{\nabla}_{X} Y-\tilde{\nabla}_{Y} X-v([X, Y]) \quad \text { for any } \quad X, Y \in \Gamma(T T M) .
$$

Locally, we obtain

$$
\begin{aligned}
& T\left(\frac{\delta}{\delta x^{j}}, \frac{\delta}{\delta x^{\imath}}\right)=R^{k}{ }_{\imath j} \frac{\partial}{\partial y^{k}} ; \quad T\left(\frac{\partial}{\partial y^{j}}, \frac{\delta}{\delta x^{\imath}}\right)=P^{k}{ }_{\imath j} \frac{\partial}{\partial y^{k}} ; \\
& T\left(\frac{\partial}{\partial y^{j}}, \frac{\partial}{\partial y^{\imath}}\right)={S^{k}}^{k}{ }_{i j} \frac{\partial}{\partial y^{k}} .
\end{aligned}
$$

Next, we denote by $T^{*}$ the torsion tensor field of $\tilde{\nabla}^{*}$. Then by using a similar formula to (3.13) we get

$$
\begin{aligned}
& T^{*}\left(\frac{\delta}{\delta x^{\jmath}}, \frac{\delta}{\delta x^{\imath}}\right)=T_{\imath}{ }^{k}{ }_{j} \frac{\delta}{\delta x^{k}} ; \quad T *\left(\frac{\partial}{\partial y^{\jmath}}, \frac{\delta}{\delta x^{\imath}}\right)=C_{\imath}{ }^{k}{ }_{j} \frac{\delta}{\delta x^{k}} ; \\
& T *\left(\frac{\partial}{\partial y^{\jmath}}, \frac{\partial}{\partial y^{\imath}}\right)=0 .
\end{aligned}
$$

Thus our assertion follows from (3.14) and (3.15).

Therefore, starting with a Finsler connection on $F^{n}$ we obtain two general Finsler connections on TTM mentioned above. Now, we consider a general Finsler connection $G F C=(N, v, \tilde{\nabla})$ on $T T M$ with respect to the projection morphism $v$ of TTM to VTM. Then we define a linear connection $\nabla$ on VTM by

$$
\nabla_{X} V=v \tilde{\nabla}_{X} V \quad \text { for any } \quad X \in \Gamma(T T M), \quad V \in \Gamma(V T M),
$$

and obtain a Finsler connection $F C=(N, \nabla)$ on $F^{n}$. However, the vertical general Finsler connection induced by $F C$ does not coincide in general with the initial 
GFC. With respect to this problem, by (3.1) and (3.16) we easily obtain

THEOREM 3.3. The vertical general Finsler connection induced by FC coincides with the initzal general Finsler connection GFC if and only if we have:

(i) the distribution VTM is parallel with respect to $\tilde{\nabla}$, i.e., we have

$$
\tilde{\nabla}_{X} V \in \Gamma(V T M) \quad \text { for any } X \in \Gamma(T T M) \text { and } V \in \Gamma(V T M) \text {, }
$$

(ii) each section of $N$ is parallel with respect to $\tilde{\nabla}$, i.e., we have

$$
\tilde{\nabla}_{X} H=0 \text { for any } X \in \Gamma(T T M) \text { and } H \in \Gamma(N) \text {. }
$$

Remark 3.1. From the above study we can conclude that Finsler connections on a Finsler space are too particular geometrical objects. The set of Finsler connections on $F^{n}$ is in a one to one correspondence with the set of general Finsler connections on TTM satisfying the very strong conditions of Theorem 3.3. This explains (in a way) why in many cases more conditions imposed to a Finsler connection imply the Riemannian case.

In particular we can consider the Cartan connection, Berwald connection, Rund connection, etc., and investigate properties of horizontal and vertical general Finsler connections induced by them on TTM. It is not the purpose of the present paper to perform such a study. However, we note a property for the Cartan connection.

By means of the Riemannian metric $g$ on $V T M$ we define a Riemannian metric on the vector bundle TTM similar to the Sasaki one and denote it also by $g$, that is

$$
d s^{2}=g_{\imath j}(x, y) d x^{\imath} d x^{\jmath}+g_{\imath j}(x, y) \delta y^{i} \delta y^{\jmath},
$$

where $\delta y^{\imath}=d y^{2}+N^{i}{ }_{j}(x, y) d x^{j}$. Then the vertical and horizontal general Finsler connections on $F^{n}$ corresponding to the Cartan connection satisfy

$$
\tilde{\nabla}_{X} g=0 ; \quad \tilde{\nabla}^{*}{ }_{X} g=0 \quad \text { for any } \quad X \in \Gamma(T T M)
$$

and

$$
T(v Y, v Z)=T^{*}(h Y, h Z)=0 \quad \text { for any } \quad Y, Z \in \Gamma(T T M) .
$$

Thus it is possible to get more results on geometry of $F^{n}$ via both general Finsler connections induced by Cartan connection. By this method we can perform invariant computations which certainly in Finsler geometry are welcome.

Remark 3.2. If we consider the general connection $\tilde{\nabla}+\tilde{\nabla}^{*}$ on $T T M$, it becomes affine, because $v+h=I_{T T M}$ and its torsion tensor coincides with the ones of the initial Finsler connection on $F^{n}$ as easily seen from (3.13)-(3.15). 


\section{§4. General Finsler connections on a Finsler space.}

In the previous sections we started with a vectorial Finsler connection (or in particular a Finsler connection) on a certain Finsler vector bundle and obtained general Finsler connections on larger vector bundles. It is the purpose of this section to study general Finsler connections on a Finsler space $F^{n}$, that is, general Finsler connections on VTM.

Let $G F C=\left(N^{i}{ }_{\jmath}, F_{\jmath}{ }_{k}, C_{\jmath}{ }_{k}, P_{j}^{i}\right)$ be a general Finsler connection on VTM. Then we call GFC a general Finsler connection on the Finsler space $F^{n}$. Since in this particular case we have

$$
G_{i^{\prime}}^{2}=\frac{\partial x^{2}}{\partial x^{2 \prime}}
$$

by (2.1) and (2.8) we get

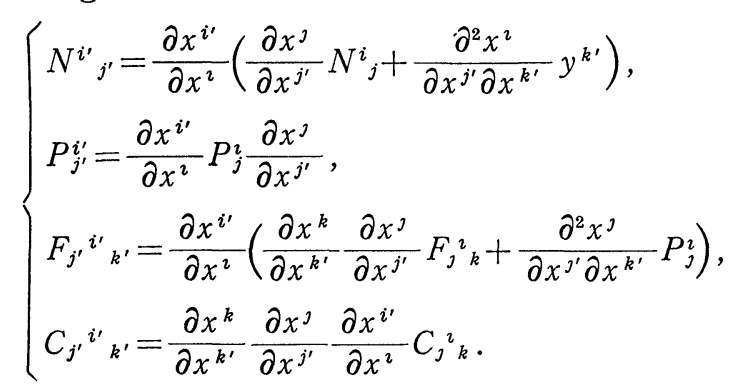

Remark 4.1. If in particular, $P$ is the identity morphism on VTM, from (4.1) we get just the transformation law for the coefficients of an usual Finsler connection on $F^{n}$ (see R. Miron [5]).

Remark 4.2. From (4.1) it follows that $P_{j}^{2}(x, y)$ and $C_{j}{ }_{k}(x, y)$ are Finsler tensor fields on $F^{n}$.

From (2.12)-(2.14) we obtain the components of the curvature tensors of a general Finsler connection on $F^{n}$ given by

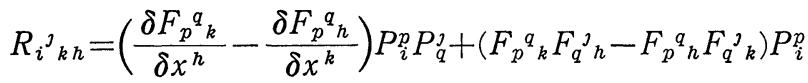

$$
\begin{aligned}
& +I_{p{ }_{k}} \Lambda_{i}{ }^{p}-I_{p}{ }^{j}{ }_{h} \Lambda_{i}{ }_{k}-R^{s}{ }_{h k} C_{p^{q}}{ }_{s} P_{i}^{p} P_{q}^{j}, \\
& P_{i}{ }_{k h}=\left(\frac{\partial F_{p}{ }^{q} k}{\partial y^{h}}-\frac{\delta C_{p}{ }^{q} h}{\delta x^{k}}\right) P_{\imath}^{p} P_{q}^{j}+\left(F_{p}{ }^{q}{ }_{k} C_{q}{ }^{j}-F_{q}{ }_{k} C_{p}{ }^{q} h\right) P_{\imath}^{p}
\end{aligned}
$$

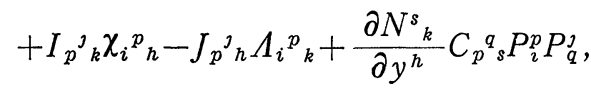

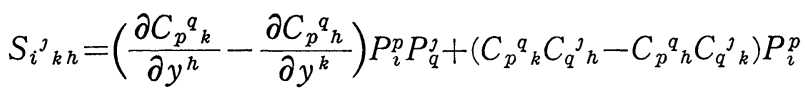

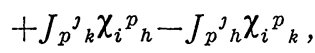


where $\Lambda_{j}{ }_{k}$ and $\chi_{j k}{ }^{2}$ are obtained from (2.11), that is we have

$$
\Lambda_{\jmath k}{ }_{k}=F_{j k}{ }_{k}-\frac{\delta P_{j}^{i}}{\delta x^{k}} ; \quad \chi_{j}{ }_{k}=C_{j}{ }^{i}-\frac{\partial P_{j}^{i}}{\partial y^{k}} .
$$

Next we shall obtain the components of torsion tensor of the general Finsler connection $G F C$ in a similar way as for the components of the torsion of a Finsler connection in Theorem 3.2. Thus, starting from a general Finsler connection $G F C=(N, P, \nabla)$ on $V T M$ we construct a general connection $G F C^{*}=$ $\left(N, L^{-1} \circ P \circ L, \nabla^{*}\right)$ on $N$ by

$$
\nabla_{X}^{*} U=L^{-1}\left(\nabla_{X} L U\right) \text { for any } X \in \Gamma(T T M) \text { and } U \in \Gamma(N),
$$

where $L$ is the isomorphism given by (3.6). Then we define the general connections $\tilde{\nabla}$ and $\tilde{\nabla}^{*}$ on $T T M$ by

$$
\begin{aligned}
& \tilde{\nabla}_{X} Y=\nabla_{X} v Y \text { and } \\
& \tilde{\nabla}_{X}{ }_{X} Y=\nabla_{X}^{*} h Y
\end{aligned}
$$

for any $X, Y \in \Gamma(T T M)$. It is easy to see that $\tilde{\nabla}$ and $\tilde{\nabla}^{*}$ are general connections with respect to $P \circ v$ and $L^{-1} \circ P \circ L \circ h$ respectively. We denote by $\widetilde{T}$ and $\tilde{T}^{*}$ the torsions of $\tilde{\nabla}$ and $\tilde{\nabla} *$ respectively and by using similar formulas as (3.13) we obtain five tensor fields:

$$
\begin{aligned}
R^{k}{ }_{i j} & =\left(\frac{\delta N_{\imath}^{h}}{\delta x^{j}}-\frac{\delta N_{\jmath}^{h}}{\delta x^{\imath}}\right) P_{h}^{k} ; \quad P^{k}{ }_{\imath j}=\frac{\partial N_{\imath}^{h}}{\partial y^{j}} P_{h}^{k}-F_{\jmath}{ }_{\imath}{ }_{\imath} ; \\
T_{\imath}{ }^{k}{ }_{j} & =F_{\imath}{ }^{k}{ }_{j}-F_{\jmath}{ }^{k}{ }_{\imath} ; \quad S^{k}{ }_{\imath j}=C_{\imath}{ }^{k}{ }_{j}-C_{\jmath}{ }^{k}{ }_{\imath} \text { and } C_{\imath}{ }^{k}{ }_{\jmath},
\end{aligned}
$$

which we call the torsions of the general Finsler connection GFC. As stated in Remark 3.2, these tensors constitutes also the torsion of the general connection $\tilde{\nabla}+\tilde{\nabla}^{*}$ on $T T M$.

Now we consider a Finsler tensor field $T_{k h}^{2 j}(x, y)$ on $F^{n}$ and look for its covariant differentiation. By a procedure performed by $T$. Otsuki in [7] we can define the $(h)$-covariant derivative of $T_{k h}^{20}$

$$
\begin{aligned}
T_{k h, s}^{i j}= & P_{p}^{i} P_{q}^{\jmath} \frac{\delta T_{l m}^{p q}}{\delta x^{s}} P_{k}^{l} P_{h}^{m}+T_{l m}^{p q} F_{p}{ }_{s} P_{q}^{j} P_{k}^{l} P_{h}^{m}+T_{l m}^{p q} F_{q}{ }_{s} P_{p}^{i} P_{k}^{l} P_{h}^{m} \\
& -T_{l m}^{p q} \Lambda_{k}{ }_{s}^{l} P_{h}^{m} P_{p}^{i} P_{q}^{\jmath}-T_{l m}^{p q} \Lambda_{h}{ }^{m}{ }_{s} P_{k}^{l} P_{p}^{i} P_{q}^{j}
\end{aligned}
$$

and its $(v)$-covariant derivative by

$$
\begin{aligned}
T_{k h \mid s}^{i j}= & P_{p}^{i} P_{q}^{j} \frac{\partial T_{l m}^{p q}}{\partial y^{s}} P_{k}^{l} P_{h}^{m}+T_{l m}^{p q} C_{p}{ }_{s}{ }_{s} P_{q}^{j} P_{k}^{l} P_{h}^{m}+T_{l m}^{p q} C_{q}{ }_{s} P_{p}^{i} P_{k}^{l} P_{h}^{m} \\
& -T_{l m}^{p q} \chi_{k}{ }_{s}{ }_{s} P_{h}^{m} P_{p}^{i} P_{q}^{j}-T_{l m}^{p q} \chi_{h}{ }^{m}{ }_{s} P_{k}^{l} P_{p}^{i} P_{q}^{\jmath} .
\end{aligned}
$$

Certainly, the $(h)$ - and $(v)$-differentiation can be performed for an arbitrary Finsler tensor field with formulas which look like (4.8) and (4.9) for more indices. 


\section{BIBLIOGRAPHY}

[1] N. ABE, General connections on vector bundles, Kodai Math. J., 8 (1985), 322-329.

[2] A. Bejancu, Geometry of Finsler subspaces I, to appear in An. Univ. "Al. I. Cuza" Iaşi, 1986.

[3] A. Bejancu, Geometry of Finsler subspaces II, to appear in Bul. Inst. Polytechnic Iaşi, 1986.

[4] M. Matsumoto, Foundations of Finsler geometry and special Finsler spaces, Kyoto Univ., 1982, unpublished.

[5] R. Miron, Introduction to the theory of Finsler spaces, Proc. Nat. Sem. Finsler Spaces, 1980, 131-184.

[6] H. NEmoto, On differential geometry of general connections, TRU Mathematics, 21 (1985), 67-94.

[7] T. ŌTsuKI, On general connections I, Math. J. Okayama Univ., 9 (1960), 99-164.

[8] T. ŌTsUKI, On general connections II, Math. J. Okyayama Univ., 10 (1961), $113-124$.

Aurel Bejancu
Institutul Politehnic
Din Iași
Iași, Romania
TOMINOSUKE OTSUKI

SCIENCE UNIVERSITY OF TOKYo

26, WAKAMIYA-CHO, ShINJUKU-KU

TOKYO 162 